\title{
Aplicación de un libro electrónico para el aprendizaje de la hoja de cálculo dirigido a estudiantes de sexto grado
}

\section{Application of an eBook for learning the spreadsheet aimed at sixth graders}

\author{
Mario José Contreras Rodríguez \\ Instituto Universitario Pedagógico Monseñor Rafael Arias Blanco \\ mcontreras@iupma.net
}

Recibido: 20/08/2016

Aceptado: 21/02/2017

Publicado: 30/06/2017

\section{RESUMEN}

Este estudio se desarrolló en el nivel de sexto grado de primaria en la asignatura de informática. Supuso exponer al estudiante a la necesidad de interactuar con un medio didáctico digital en formato de libro electrónico, para mejorar el aprendizaje de la hoja de cálculo. Se enmarca dentro de la metodología denominada Comprensión Holística de la Investigación, holotipo interactivo. Para el diagnóstico se entrevistó a docentes de la especialidad, lo cual permitió describir los problemas que afectan la enseñanza de la hoja de cálculo. Luego se elaboró una propuesta de libro electrónico, con base en estrategias cognoscitivas, la cual se aplicó a una muestra de treinta y ocho alumnos. El procedimiento incluyó la administración de una prueba de conocimientos en calidad de pretest, luego se registró el desempeño de los estudiantes mediante la observación participante y, posteriormente, se aplicó el postest. Se utilizaron técnicas de análisis cuantitativo, a través del procesamiento estadístico, y cualitativo de acuerdo al paradigma naturalista. Esto último incluyó la descripción de los eventos e interpretación apoyada por la teoría correspondiente. Los resultados, desde lo cuantitativo, sugieren que los cambios apreciados se debieron a la intervención realizada. En lo cualitativo, los alumnos realizaron ajustes en cuanto a la manera de accionar frente a un material novedoso, lo cual abarcó la lectura no lineal, resolver situaciones e interactuar con el docente y sus compañeros, como parte de un proceso de elaboración y construcción. En este sentido, las evidencias reflejaron un desarrollo en las habilidades de tipo procedimental y la capacidad de manejar información de manera fragmentaria y asociativa.

\section{PALABRAS CLAVE}

Educación tecnológica; Medios de enseñanza; Hoja de cálculo; Uso didáctico del ordenador.

\section{ABSTRACT}

The study was developed at the level of sixth grade, computer course and makes the student interact with a digital teaching aid in eBook format, to improve the learning of spreadsheet. The methodology is based on holistic research method, interactive type. The diagnosis phase included interviews to informatics teachers, which allowed describe the problems affecting the teaching of the spreadsheet. Then a proposal of eBook, based on cognitive strategies, was elaborated and applied to a sample of 38 students. The procedure included a pre-test, student performance registration by participant observation and the post-test. Quantitative analysis techniques were used, through statistical processing, and also qualitative according to naturalistic paradigm. Here events description and interpretation supported by the corresponding theory was performed. Quantitative results suggest that changes were due to the intervention, while qualitative results indicate that students made adjustments on how to work with a new teaching aid, which included the non-linear reading, having to reflect on how to resolve situations and interact with teacher and peers, as part of a process of development and construction. The evidence reflected skills development of procedural type and the ability to deal with information in a fragmentary and associative mode.

\section{KEYWORDS}

Technological education; Teaching aid; Spreadsheet; Didactic use of computer.

\section{CITA RECOMENDADA}

Contreras, M., J. (2017). Aplicación de un libro electrónico para el aprendizaje de la hoja de cálculo dirigido a estudiantes de sexto grado. RIITE. Revista Interuniversitaria de Investigación en Tecnología Educativa, 2, 13-27. Doi: http://dx.doi.org/10.6018/riite/2017/266241 
¿Qué se había investigado sobre el tema?

- Ramos, P. y Moreira, J. (2013). eBook: un dispositivo pedagógico en la enseñanza y aprendizaje de biología y geología - Un caso de estudio con estudiantes de onceavo grado, de la Escuela Básica y Secundaria de Cerco, Porto, Portugal. Este trabajo conceptualiza, ejecuta y evalúa un libro electrónico, creado de acuerdo a dos perspectivas: una orientada hacia la enseñanza y el aprendizaje, y otra centrada en indagar si el ebook podría ser considerado como un instrumento pedagógico.

- Canchignia, X. (2011). Libro electrónico de openoffice.org Calc para mejorar el aprendizaje del módulo de Hoja electrónica de cálculo en el segundo año de bachillerato del Instituto Tecnológico Victoria Vásconez Cuvi, Latacunga. Ecuador. Este trabajo pretende promover las funcionalidades de la hoja de cálculo en ambiente de código abierto, a través de un libro electrónico, el cual se constituye, además, en una alternativa que despierte la motivación de los educandos frente a una didáctica tradicional, carente de recursos actualizados, de acuerdo al contexto que escribe el autor.

- Lai, H. y Li, M. (2013). Gestión del Conocimiento y e-Learning. Un estudio de los comportamientos de los estudiantes universitarios en el uso de libros electrónicos en Hong Kong.

\section{Principales aportaciones del artículo}

- Aporta información sobre el empleo del libro electrónico como recurso a emplear en distintas áreas del saber en la escuela primaria.

- Aporta datos sobre el empleo del libro electrónico mediante estrategias cognoscitivas, las cuales pueden ubicarse dentro de una tendencia postconductista del desarrollo de software educativo, ya que, con este proyecto, se promovió un ambiente de aprendizaje donde se valoraron el rol activo tanto del estudiante como del docente, los procesos y las interacciones alumno-material, alumnoalumno y alumno-docente.

\section{Futuras líneas de investigación}

- Medios didácticos digitales en la escuela primaria.

- El eBook como dispositivo pedagógico para la implementación de estrategias cognoscitivas.

- El libro electrónico como herramienta para el desarrollo de la capacidad asociativa y la lectura no lineal.

\section{INTRODUCCIÓN}

Los desafíos actuales del docente en educación informática se centran en cómo lograr que el aprendizaje de esta disciplina, más que un fin en sí mismo, facilite la integración con otras áreas del saber y promueva en el educando habilidades para descartar información irrelevante, analizar y sintetizar la información pertinente y asimilar el nuevo conocimiento (Casado, 2013). En este sentido, conviene exponer al estudiante a la situación de resolver una actividad o un problema vinculado con la vida cotidiana (mediado por el ordenador), donde se haga necesario para su solución eficiente, la integración de contenidos de otras asignaturas del currículum (Miranda, Pirez y García, 2012).

Igualmente, que pueda activar sus estructuras cognitivas, lo cual le facilite recordar, describir o demostrar conocimientos o experiencias previas que le sean relevantes, así como integrar los nuevos conocimientos a dichas estructuras cognoscentes. También que sea capaz de compartir sus experiencias entre pares. En fin, que sea un sujeto activo, dentro del marco de un nuevo paradigma post-industrial, el cual se concreta en un sistema instruccional basado en el aprendizaje (Reigeluth, 2012).

Con base en las prestaciones que ofrece, surge el libro electrónico como una alternativa a la versión impresa, el cual destaca por sus posibilidades en cuanto a despertar y mantener el interés de los estudiantes, a través de las diversas maneras de cómo presentar la información, 
organizar y relacionar los contenidos, así como evaluar los conocimientos y las habilidades que se adquieren (Marquès, 2011).

Ya que, en un principio, hubo confusión acerca de su verdadera naturaleza al ser un término usado en forma ambigua para denominar textos electrónicos (e-text), libros formateados en archivos de texto o escaneados, se consideró un formato que precisaba mejoras para hacerlo realmente útil y accesible a todo público (Elizondo, 2006). Hoy quizás, pocos cuestionen su potencialidad como medio didáctico digital. Sin embargo, aún persisten aspectos sin resolver, como el deslumbramiento de la pantalla y la tensión ocular que puede generar, los cuales son impedimentos para una lectura adecuada y una de las razones para que muchos estudiantes prefirieran libros impresos sobre libros electrónicos. Se espera que la tecnología de e-Ink podría mejorar y resolver estos problemas (Lai y Li, 2013).

La decisión de investigar en este campo obedeció a situaciones observadas en el contexto de la clase de informática en sexto grado del colegio San Agustín El Marqués, en Caracas, relacionadas con la pertinencia de los textos de informática, el desempeño de los educandos y la percepción acerca de los medios didácticos digitales, de acuerdo a la opinión de docentes de informática.

La Epistemología Genética de Piaget (1991) tuvo implicación toda vez que se trató con niños ubicados en la etapa de operaciones concretas, quienes, por lo general, presentan dificultades situarse en el campo de lo abstracto. Por lo tanto, la situación de aprendizaje debía contemplarse en términos de un equilibrio entre la cantidad de conocimiento declarativo que ofreciera el medio y la nueva información adquirida por el alumno. Esto suponía, además, que el docente pudiera introducir el vocabulario técnico de manera progresiva, y así el niño lograra, con mayor facilidad, que el material le fuera significativo.

Finalmente, la propuesta supuso que el educando aprendiera, desde una perspectiva cognoscitiva, un conjunto de estrategias en las áreas de adquisición de conocimiento, estudio y resolución de problemas. Con este fin se elaboró una taxonomía de estrategias cognoscitivas (Poggioli, 2009), la cual sirvió para codificar los datos recogidos durante la interacción de los estudiantes con el libro electrónico.

\section{MÉTODO}

El enfoque metodológico se basó en la Comprensión Holística de la Investigación (Hurtado, 2012), la cual propone abordar la solución a un problema de investigación a través de técnicas mixtas de recogida y de análisis datos.

Se trabajó con un diseño transeccional, de campo, con el propósito de modificar una situación exclusiva del contexto de la clase de informática de sexto grado.

Se trabajó con treinta y ocho (38) estudiantes, de edades comprendidas entre diez y doce años, escogidos mediante muestreo no probabilístico e incidental, de una población de 160 sujetos.

Los instrumentos de recolección de datos (una guía de entrevistas, una prueba de conocimientos y un cuestionario a los estudiantes) se validaron mediante el juicio de expertos. Todos los instrumentos obtuvieron un índice de validez mayor al 0.70 . 


\subsection{Objetivos}

General: Aplicar un libro electrónico basado en estrategias cognoscitivas para el aprendizaje de la hoja de cálculo.

Específicos:

- Describir los problemas más comunes que afectan a la enseñanza de la hoja de cálculo en sexto grado, de acuerdo a la opinión de docentes de la especialidad.

- Diseñar un libro electrónico contentivo de estrategias que aborden los problemas detectados.

- Registrar el desempeño de los educandos durante su interacción con el libro electrónico.

- Verificar los cambios que tuvieron lugar posterior a la aplicación del libro electrónico.

\subsection{Procedimiento}

Al comienzo del estudio se realizó un diagnóstico para conocer los problemas que afectan la enseñanza de la informática en sexto grado, para lo cual se entrevistó a cuatro docentes de de infomática. Las grabaciones se transcribieron a formato compatible con el software Atlas-Ti. Se realizó la categorización, codificación, tabulación, análisis e interpretación de los datos.

Los resultados del diagnóstico sirvieron de insumo para una revisión documental con el fin de determinar un diseño instruccional idóneo. Se eligió la Metodología Dinámica para el Desarrollo de un Software Educativo de Arias, López y Rosario (2002), el cual propone una instrucción dirigida a desarrollar en el individuo estrategias que faciliten la selección, percepción, procesamiento y recuperación de la información; y concibe al ordenador como un medio dinámico, dada su capacidad multimedia.

El proceso abarca cuatro fases: Diseño Educativo, Producción, Realización e Implementación; y un eje transversal de Evaluación (Figura 1).

Luego, se realizó la planeación de la intervención. Se diseñaron los objetivos, actividades y estrategias que conformaron el libro electrónico. El prototipo fue sometido al juicio de dos expertos: un docente de matemáticas, para evaluar el contenido, y otro de informática, para evaluar aspectos relacionados con el diseño del software. Con este fin se aplicó el Instrumento de Evaluación para un Software Educativo.

Se aplicó la prueba pretest. Luego, se realizó la observación sistemática del desempeño de los educandos mientras interactuaron con el libro electrónico, la cual también sirvió para modificar, sobre la marcha, aspectos de la propuesta que así lo ameritaran (Hurtado, ob. cit.). De esta manera, se recabaron los datos de situaciones, comportamientos observables, testimonios de los alumnos, entre otros aspectos. 


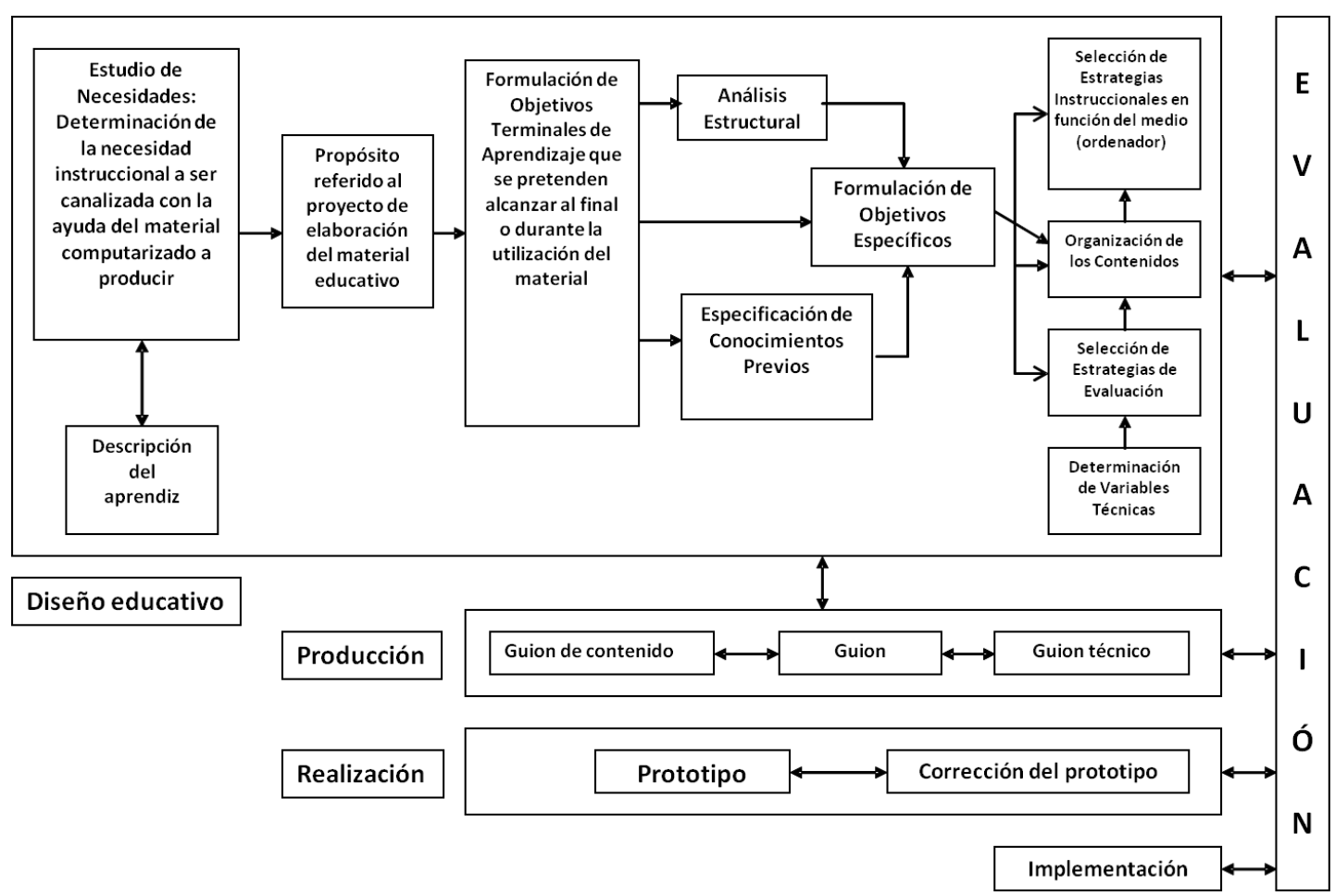

Figura 1. Metodología Dinámica para el Desarrollo de Software Educativo propuesta por Arias, López y Rosario (2002)

Posteriormente, se aplicó la prueba de conocimientos (pos-test). Finalmente, se pidió a los estudiantes que completaran un cuestionario mixto, en el cual pudieron expresar su opinión sobre aspectos generales del trabajo con el libro electrónico y sobre el desempeño del docente. El instrumento se envió mediante el servicio de encuestas basado en la web denominado Survey Monkey.

Se elaboró una matriz de análisis cualitativo. Cada código se asoció a una descripción del evento y se confirmó con una interpretación de acuerdo a la teoría revisada. Se aplicó estadística descriptiva e inferencial para realizar comparaciones entre el pre-test y el pos-test.

\section{RESULTADOS}

\subsection{Diagnóstico}

En el nivel textual, cada entrevista se definió como un documento primario (DP). Del total de unidades de análisis (citas en Atlas-Ti) contentivas de opiniones, valoraciones, conductas, secuencias, entre otros (Strauss y Corbin 2002, citados por Hurtado, 2012) se realizó una primera reducción que permitió asignar cincuenta y seis (56) códigos extraídos del texto, en cuyo caso toman el nombre de "código en vivo" (Strauss y Corbin 2002, citados por Hurtado, ob. Sit.). A través de una tabla descriptiva de frecuencias se puede observar cuáles fueron las categorías emergentes y su frecuencia en cada DP (Tabla 1). 
Tabla 1. Descripción de frecuencias por categoría

07 Enseñanza a través de la práctica

07 Aprendizaje por descubrimiento

07 Definir teoría

07 Técnica de la Preguntas

07 Trabajo colaborativo

08 Planificación en función del diagnóstico y del objetivo

08 Planificación por lapso

08 Resolver problemas

09 Buen desempeño general

09 Importancia de la motivación

10 Poca capacidad de abstracción

10 Si realizan abstracciones

11 Facilitador

11 No especifica rol docente

11 Realimentación

12 Facilita la transferencia

12 No hay transferencia

13 Textos poco adaptados

14 Debe tener un formato y estructura

15 Biblioteca digital como apoyo a las estrategias docentes

15 Centro cultural

16 Correo electrónico

16 Guía de ejercicios

16 Impresos

16 Video proyector

16 Videos

17 Importancia de la interactividad

17 Amplía las posibilidades de enseñanza

17 Importancia de la capacidad multimedia

17 Recurso didáctico enlazado con recursos remotos

18 Debe incluir hipervínculos

19 Importancia de la transversalidad

19 Prevalece la práctica sobre lo conceptual

19 Sí puede enseñarse teoría y práctica simultáneamente

20 Breve teoría y ejercicios

20 Favorece enfoque conductista en los niños

20 Materiales carecen de teoría que los fundamente

20 Materiales desactualizados

20 No hay un programa oficial para informática

21 Aritmética

21 Características del formato y utilidad de las celdas

21 Entender Excel como un plano de dos dimensiones

21 Estadística

21 Fórmulas, funciones, gráficos

21 Números decimales y binarios

21 Referencias

22 No se observa uso de nuevo vocabulario

22 Sí se observa uso de nuevo vocabulario

$23 \mathrm{La}$ algoritmia permite evidenciar el pensamiento reflexivo

$23 \mathrm{Si}$ se observa pensamiento reflexivo

24 Competencia pero no colaboración

24 Se observa logro colectivo

DP 1 DP 2 DP 3 DP 4 Total

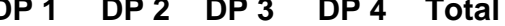

$\begin{array}{llllll}0 & 3 & 1 & 0 & 4\end{array}$

$\begin{array}{lllll}1 & 0 & 0 & 0 & 1\end{array}$

$\begin{array}{lllll}1 & 0 & 0 & 0 & 1\end{array}$

$\begin{array}{lllll}0 & 0 & 0 & 1 & 1\end{array}$

$\begin{array}{lllll}0 & 1 & 0 & 0 & 1\end{array}$

$\begin{array}{lllll}0 & 0 & 1 & 1 & 2\end{array}$

$\begin{array}{lllll}0 & 1 & 0 & 0 & 1\end{array}$

$\begin{array}{lllll}3 & 0 & 0 & 0 & 3\end{array}$

$\begin{array}{llllll}0 & 1 & 1 & 0 & 2\end{array}$

$\begin{array}{lllll}1 & 0 & 0 & 1 & 2\end{array}$

$\begin{array}{lllll}1 & 0 & 0 & 0 & 1\end{array}$

$\begin{array}{lllll}0 & 1 & 1 & 0 & 2\end{array}$

$\begin{array}{lllll}1 & 0 & 0 & 0 & 1\end{array}$

$\begin{array}{lllll}0 & 1 & 1 & 1 & 3\end{array}$

$\begin{array}{lllll}1 & 0 & 0 & 0 & 1\end{array}$

$\begin{array}{lllll}0 & 1 & 1 & 0 & 2\end{array}$

$\begin{array}{lllll}1 & 0 & 0 & 0 & 1\end{array}$

$\begin{array}{lllll}1 & 1 & 1 & 1 & 4\end{array}$

$\begin{array}{lllll}2 & 1 & 1 & 1 & 5\end{array}$

0 
24 Se observa mucho individualismo en los alumnos

25 Poco respeto por las ideas del otro

25 Se observa respeto por las ideas del otro

25 Solo hay respeto si hay convergencia de ideas

$\begin{array}{rccccr} & 1 & 0 & 0 & 0 & 1 \\ & 1 & 1 & 0 & 0 & 2 \\ & 0 & 0 & 2 & 1 & 3 \\ \text { Totales } & 1 & 0 & 0 & 0 & 1 \\ & 29 & 25 & 26 & 21 & 101\end{array}$

Al relacionar los conceptos manejados en la investigación con la información objeto de análisis, se configuró la matriz de categorías que se muestra en la Tabla 2.

Tabla 2. Matriz de Categorías

\begin{tabular}{|c|c|c|c|}
\hline Evento & Dimensiones & Indicios & Categoría \\
\hline \multirow{16}{*}{$\begin{array}{l}\text { Aplicación } \\
\text { del libro } \\
\text { electrónico }\end{array}$} & \multirow{10}{*}{$\begin{array}{l}\text { Estrategias } \\
\text { Cognitivas } \\
\text { (Poggioli) }\end{array}$} & $\begin{array}{l}\text { Enfoque } \\
\text { teórico }\end{array}$ & $\begin{array}{l}07 \text { Enseñanza a través de la práctica } \\
07 \text { Aprendizaje por descubrimiento } \\
07 \text { Definir teoría } \\
07 \text { Técnica de la Pregunta } \\
\text { 07 Trabajo colaborativo }\end{array}$ \\
\hline & & $\begin{array}{l}\text { Resolución de } \\
\text { problemas } \\
\text { (Hoja de } \\
\text { cálculo) }\end{array}$ & $\begin{array}{l}12 \text { Facilita la transferencia } \\
12 \text { No hay transferencia }\end{array}$ \\
\hline & & $\begin{array}{l}\text { Rol del } \\
\text { docente }\end{array}$ & $\begin{array}{l}11 \text { Facilitador } \\
11 \text { No especifica rol docente } \\
11 \text { Realimentación }\end{array}$ \\
\hline & & $\begin{array}{l}\text { Capacidad de } \\
\text { Abstracción }\end{array}$ & $\begin{array}{l}10 \text { Poca capacidad de abstracción } \\
10 \text { Si realizan abstracciones }\end{array}$ \\
\hline & & Motivación & $\begin{array}{l}9 \text { Buen desempeño general } \\
9 \text { Importancia de la motivación }\end{array}$ \\
\hline & & $\begin{array}{l}\text { Organización } \\
\text { de los } \\
\text { contenidos }\end{array}$ & 13 Textos poco adaptados \\
\hline & & Planificación & $\begin{array}{l}\text { 08 Planificación en función del diagnóstico y del } \\
\text { objetivo } \\
\text { 08 Planificación por lapso } \\
\text { 08 Resolver problemas }\end{array}$ \\
\hline & & \multirow{3}{*}{$\begin{array}{l}\text { Recursos a } \\
\text { utilizar }\end{array}$} & $\begin{array}{l}15 \text { Biblioteca digital como apoyo y complemento a las } \\
\text { estrategias docentes } \\
15 \text { Biblioteca digital como centro cultural }\end{array}$ \\
\hline & & & $\begin{array}{l}16 \text { Correo electrónico } \\
16 \text { Guía de ejercicios } \\
16 \text { Impresos } \\
16 \text { Video proyector } \\
16 \text { Videos } \\
\end{array}$ \\
\hline & & & $\begin{array}{l}17 \text { Importancia de la interactividad } \\
17 \text { Amplia las posibilidades de enseñanza } \\
17 \text { Importancia de la capacidad multimedia } \\
17 \text { Recurso didáctico enlazado con recursos remotos. }\end{array}$ \\
\hline & \multirow{6}{*}{$\begin{array}{l}\text { Diseño, } \\
\text { Concordancia, } \\
\text { Coherencia }\end{array}$} & \multirow{4}{*}{$\begin{array}{l}\text { Desarrollo } \\
\text { Teórico- } \\
\text { práctico }\end{array}$} & 13 Textos poco adaptados \\
\hline & & & 14 Debe tener un formato y estructura \\
\hline & & & 18 Debe incluir hipervínculos \\
\hline & & & $\begin{array}{l}19 \text { Importancia de la transversalidad } \\
19 \text { Prevalece la práctica sobre lo conceptual } \\
19 \text { Sí puede enseñarse teoría y práctica } \\
\text { simultáneamente }\end{array}$ \\
\hline & & \multirow[t]{2}{*}{$\begin{array}{l}\text { Secciones del } \\
\text { libro } \\
\text { electrónico }\end{array}$} & $\begin{array}{l}20 \text { Breve teoría y ejercicios } \\
20 \text { Favorece enfoque conductista en los niños } \\
20 \text { Materiales carecen de teoría que los fundamente } \\
20 \text { No hay un programa oficial para informática. }\end{array}$ \\
\hline & & & 20 Materiales poco actualizados \\
\hline
\end{tabular}




\begin{tabular}{|c|c|c|}
\hline & $\begin{array}{l}\text { Herramientas } \\
\text { de la hoja de } \\
\text { cálculo }\end{array}$ & $\begin{array}{l}21 \text { Aritmética } \\
21 \text { Características del formato y utilidad de las celdas } \\
21 \text { Entender Excel como un plano de dos } \\
\text { dimensiones } \\
21 \text { Estadística } \\
21 \text { Fórmulas, funciones, gráficos } \\
21 \text { Números decimales y binarios } \\
21 \text { Referencias }\end{array}$ \\
\hline \multirow{6}{*}{$\begin{array}{l}\text { Aprendizaje } \\
\text { significativo }\end{array}$} & $\begin{array}{l}\text { Manejo de } \\
\text { vocabulario }\end{array}$ & $\begin{array}{l}22 \text { No se observa uso de nuevo vocabulario } \\
22 \text { Si se observa uso de nuevo vocabulario }\end{array}$ \\
\hline & $\begin{array}{l}\text { Pensamiento } \\
\text { reflexivo }\end{array}$ & $\begin{array}{l}23 \text { La algoritmia permite evidenciar el pensamiento } \\
\text { reflexivo } \\
23 \text { Si se observa pensamiento reflexivo }\end{array}$ \\
\hline & \multirow{2}{*}{$\begin{array}{l}\text { Solución de } \\
\text { problemas en } \\
\text { conjunto }\end{array}$} & $\begin{array}{l}24 \text { Competencia pero no colaboración } \\
24 \text { Se observa logro colectivo }\end{array}$ \\
\hline & & 24 Se observa individualismo en los alumnos \\
\hline & \multirow{2}{*}{$\begin{array}{l}\text { Respeto a las } \\
\text { ideas del otro }\end{array}$} & 25 Poco respeto por las ideas del otro \\
\hline & & $\begin{array}{l}25 \text { Se observa respeto por las ideas del otro } \\
25 \text { Solo hay respeto si hay convergencia de ideas }\end{array}$ \\
\hline
\end{tabular}

\subsection{Propuesta}

La planeación de la intervención se concretó a través de una propuesta de libro electrónico articulada en torno a objetivos de duración variable (Briones, 1991, citado por Hurtado, 2012). El guion de contenido resume la estructura general del libro electrónico, la cual se muestra en la Figura 2.

Destaca en este esquema la línea de producción del prototipo, la cual está concebida para ser desarrollada por docentes con conocimientos medios de informática. Se emplearon herramientas ofimáticas para la creación de un primer borrador, el cual se guardó en formato PDF y luego fue convertido a formato libro electrónico, provisto de tecnología page flipping, mediante el software aXMag.

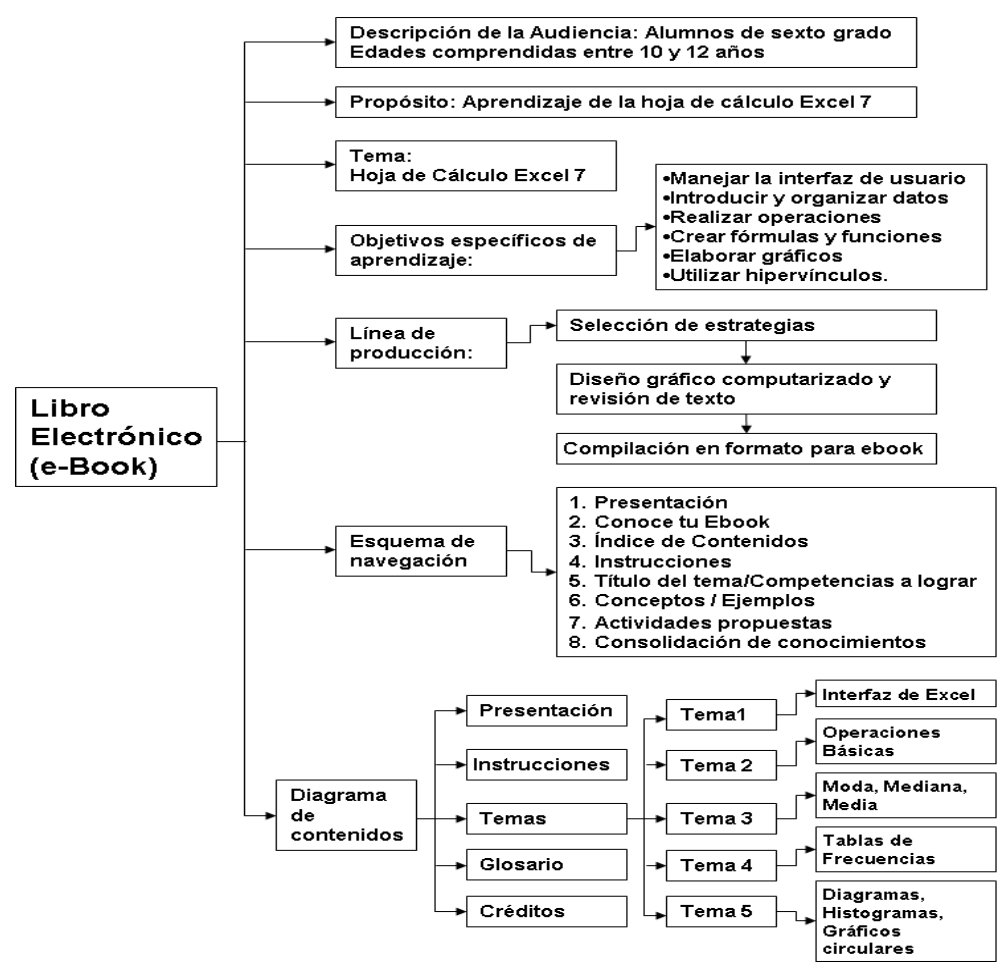

Figura 2. Guion de Contenido 


\subsection{Observación del desempeño}

Con el fin de interpretar la información obtenida durante la aplicación de la propuesta, se establecieron categorías de análisis, códigos e indicadores (Tabla 3), para facilitar su identificación en los cuadros de observaciones. Se creó una tabla de doble entrada, con los sujetos en el eje vertical y las categorías emergentes en el eje horizontal, con base en el modelo de Louis de Vivas (1994).

Tabla 3. Sistema de Categorías de Análisis

\begin{tabular}{|c|c|c|c|}
\hline Categorías & Dimensión & Código & Indicador \\
\hline \multirow{4}{*}{ Cognición } & $\begin{array}{l}\text { Organización de } \\
\text { la información }\end{array}$ & $\begin{array}{l}\text { GNC } \\
\text { RPS } \\
\text { ADV }\end{array}$ & $\begin{array}{l}\text { Genera notas, agrupa y clasifica datos. } \\
\text { Respeta los pasos y secuencias. } \\
\text { Aclara dudas y continua con el material. }\end{array}$ \\
\hline & $\begin{array}{l}\text { Elaboración y } \\
\text { Construcción }\end{array}$ & $\begin{array}{l}\text { RPC } \\
\text { DM } \\
\text { ELV }\end{array}$ & $\begin{array}{l}\text { Realiza producciones de calidad. } \\
\text { Demuestra motivación. } \\
\text { Elabora verbalmente (anticipa, predice, } \\
\text { elabora hipótesis, realiza / responde } \\
\text { preguntas). }\end{array}$ \\
\hline & Comprensión & $\begin{array}{l}\text { LRS } \\
\text { ECI } \\
\text { REA }\end{array}$ & $\begin{array}{l}\text { Lee respetando signos de puntuación. } \\
\text { Evidencia comprensión en la } \\
\text { intervención. } \\
\text { Provee realimentación. }\end{array}$ \\
\hline & $\begin{array}{l}\text { Recuperación de } \\
\text { la información }\end{array}$ & $\begin{array}{l}\mathrm{RE} \\
\mathrm{RCA}\end{array}$ & $\begin{array}{l}\text { Evidencia recuperación efectiva. } \\
\text { Recupera información con ayuda. }\end{array}$ \\
\hline Metacognición & & $\begin{array}{l}\text { BS } \\
\text { BAS } \\
\text { GNC }\end{array}$ & $\begin{array}{l}\text { Busca soluciones a los problemas. } \\
\text { Busca alternativas de solución. } \\
\text { Clasificar, tomar notas, agrupar. }\end{array}$ \\
\hline Socialización & & $\begin{array}{l}\text { TC } \\
\text { CMS }\end{array}$ & $\begin{array}{l}\text { Ayuda al compañero. } \\
\text { Confianza para manifestar sentimientos }\end{array}$ \\
\hline \multirow{2}{*}{$\begin{array}{l}\text { Aprendizaje } \\
\text { significativo }\end{array}$} & $\begin{array}{l}\text { Aprendizaje } \\
\text { significativo en } \\
\text { ascenso }\end{array}$ & ASA & $\begin{array}{l}\text { Enlaza con conocimientos previos de } \\
\text { manera parcial. }\end{array}$ \\
\hline & $\begin{array}{l}\text { Aprendizaje } \\
\text { significativo } \\
\text { completado }\end{array}$ & ASC & $\begin{array}{l}\text { Enlaza con conocimientos previos de } \\
\text { manera efectiva. }\end{array}$ \\
\hline
\end{tabular}

Finalmente, se diseñó una matriz de análisis de la información en la que se presentan los códigos utilizados, la descripción del evento y la interpretación apoyada por la teoría correspondiente (Tabla 4). 
Tabla 4. Matriz de análisis de la información (Resumen de las observaciones)

\begin{tabular}{|c|c|c|}
\hline Código & Descripción & Interpretación \\
\hline $\begin{array}{l}\text { RPS } \\
\text { ORG }\end{array}$ & $\begin{array}{l}\text { Durante el desarrollo de las actividades } \\
\text { correspondientes al tema } 1 \text { (Conoce la } \\
\text { hoja de cálculo), la mayoría de los } \\
\text { estudiantes respetó los pasos y } \\
\text { secuencias que establece el libro } \\
\text { electrónico. Algunos evidenciaron } \\
\text { dificultad al realizar esta actividad. El } \\
\text { docente recalcó la importancia de } \\
\text { llegar a tiempo y dio libertad para } \\
\text { completar las actividades al ritmo } \\
\text { propio. }\end{array}$ & $\begin{array}{l}\text { Las estrategias de motivación y apoyo persiguen, } \\
\text { entre otros propósitos, enfocar la atención, mantener } \\
\text { la concentración, manejar la ansiedad y generar un } \\
\text { clima de respeto a las normas. Para Poggioli (ob. } \\
\text { cit.), estas estrategias persiguen identificar } \\
\text { obstáculos internos, como actitudes e interferencias } \\
\text { que puedan obstaculizar el desarrollo adecuado de } \\
\text { las actividades. }\end{array}$ \\
\hline EVD & $\begin{array}{l}\text { Hubo evidencia de } \\
\text { ubicar y aplicar } \\
\text { opciones. }\end{array}$ & $\begin{array}{l}\text { La interacción con un nuevo medio didáctico puede } \\
\text { generar ciertas dificultades al inicio, debido a que el } \\
\text { alumno no está habituado a manejar materiales } \\
\text { instruccionales; oye pero no comprende las } \\
\text { indicaciones o no logra concentrarse. Al acumular } \\
\text { experiencias de tipo procedimental, el educando logra } \\
\text { desarrollar la habilidad para interactuar con el } \\
\text { material y construye esquemas que le guían en la } \\
\text { ejecución de tareas similares (Poggioli, ob. cit.). }\end{array}$ \\
\hline $\begin{array}{l}\text { RPS } \\
\text { TC } \\
\text { ELV }\end{array}$ & $\begin{array}{l}\text { Se observó que los alumnos que } \\
\text { demostraron organización, al respetar } \\
\text { los pasos, fueron con frecuencia } \\
\text { quienes también elaboraron de manera } \\
\text { verbal, participaron activamente en la } \\
\text { clase y ayudaron a otros compañeros }\end{array}$ & $\begin{array}{l}\text { Con el aprendizaje colaborativo el alumno trabaja a } \\
\text { su propio ritmo, pero motivado a buscar soluciones } \\
\text { en conjunto, consciente de las dificultades propias y } \\
\text { del compañero. Esto favorece el sentido de cohesión } \\
\text { al compartir metas comunes, lo cual propicia el } \\
\text { crecimiento y desarrollo de cada individuo, a través } \\
\text { de las relaciones interpersonales y la productividad } \\
\text { (Calzadilla, 2002). Al aportar ayuda al compañero de } \\
\text { manera espontánea, el alumno elabora verbalmente y } \\
\text { evidencia recuperación efectiva de la información. }\end{array}$ \\
\hline $\begin{array}{l}\text { ELV } \\
\text { APA } \\
\text { APT }\end{array}$ & $\begin{array}{l}\text { Destaca la intervención de una niña, } \\
\text { quien durante la creación de listas } \\
\text { comentó que el programa Excel le } \\
\text { sería útil para elaborar su horario de } \\
\text { clases. }\end{array}$ & $\begin{array}{l}\text { Evidencia de enlace del conocimiento adquirido con } \\
\text { otra necesidad académica, lo cual sugiere una } \\
\text { aproximación a la transferencia. Gagné }(1979, \text { p. } 41) \\
\text { señala que, al seleccionar de manera adecuada los } \\
\text { eventos a presentar en un material, se facilita la } \\
\text { transferencia. }\end{array}$ \\
\hline RPS & $\begin{array}{l}\text { Actividades correspondientes al Tema } \\
\text { 2: Creación de fórmulas. Se realizó en } \\
\text { dos sesiones, la primera para la } \\
\text { construcción de la tabla y la segunda } \\
\text { para los cálculos y verificaciones. Se } \\
\text { inició con la lectura (grupal). El proceso } \\
\text { supuso analizar el espacio de la hoja } \\
\text { de cálculo y distribuir los elementos } \\
\text { según lo indicaba el libro electrónico. } \\
\text { Los estudiantes que mostraban rezago } \\
\text { completaron la actividad pendiente e } \\
\text { iniciaron el nuevo tema }\end{array}$ & $\begin{array}{l}\text { Las estrategias cognitivas de organización se ven } \\
\text { favorecidas por la manera cómo se presenta la } \\
\text { información (imágenes, conceptos, procedimientos), } \\
\text { lo cual induce al alumno a accionar de manera } \\
\text { estratégica, mediante actividades que hacen posible } \\
\text { organizar, retener y recuperar la información que se } \\
\text { desea asimile (aprender desde una perspectiva } \\
\text { cognoscitiva (Poggioli, 2009). El interés demostrado } \\
\text { refleja motivación por efecto del material, pero } \\
\text { también depende de la responsabilidad del } \\
\text { educando. }\end{array}$ \\
\hline ELV & $\begin{array}{l}\text { Tres alumnos realizaron preguntas al } \\
\text { docente antes de completar la tabla, } \\
\text { para confirmar si lo que habían leído } \\
\text { era correcto. }\end{array}$ & $\begin{array}{l}\text { El realizar preguntas para no cometer } \\
\text { equivocaciones, lejos de ser un indicador negativo, } \\
\text { refleja motivación e interés en lograr producciones de } \\
\text { calidad. }\end{array}$ \\
\hline BS & $\begin{array}{l}\text { Se instó a los alumnos a buscar una } \\
\text { solución alternativa para realizar las } \\
\text { verificaciones de los descuentos. Esta } \\
\text { variante suponía interpretar una } \\
\text { ilustración de ejemplo. }\end{array}$ & $\begin{array}{l}\text { Dentro de las estrategias de estudio y ayudas } \\
\text { anexas, el aprendizaje por descubrimiento se puede } \\
\text { lograr al solicitar a los estudiantes que descifren una } \\
\text { tarea en lugar de dar la "receta". Esto persigue } \\
\text { promover el pensamiento crítico Poggioli (2009). }\end{array}$ \\
\hline ASC & $\begin{array}{l}\text { Se evidenció mejora general en la } \\
\text { lectura de instrucciones y realización } \\
\text { de actividades, en comparación a } \\
\text { sesión anterior. Hubo } 4 \text { alumnos que } \\
\text { iniciaron el Tema } 3 .\end{array}$ & $\begin{array}{l}\text { Ante el usual rechazo que supone la interacción con } \\
\text { materiales que incluyen requerimientos de } \\
\text { decisiones, disciplina y comprensión, la teoría } \\
\text { cognitiva propone un cambio hacia procedimientos } \\
\text { para dirigir el procesamiento y la interacción de los } \\
\text { estudiantes con el sistema de diseño de instrucción } \\
\text { (Merril, Kowalis, y Wilson, 1981, citados por Ertmer y } \\
\text { Newby, 1993). }\end{array}$ \\
\hline
\end{tabular}




\subsection{Prueba de Conocimientos}

Los resultados del pretest estuvieron entre 1 y 3 (de un máximo de 5), con una media de 1,2; mientras que en el postest fueron de 2 a 4, con una media de 2,8. Sólo 3 alumnos tuvieron ganancia cero, es decir, obtuvieron la misma calificación en ambas pruebas. La diferencia entre ambos momentos arroja una ganancia en la media del 31,1\%, con una ganancia mínima del $20 \%$ y una ganancia máxima del $60 \%$.

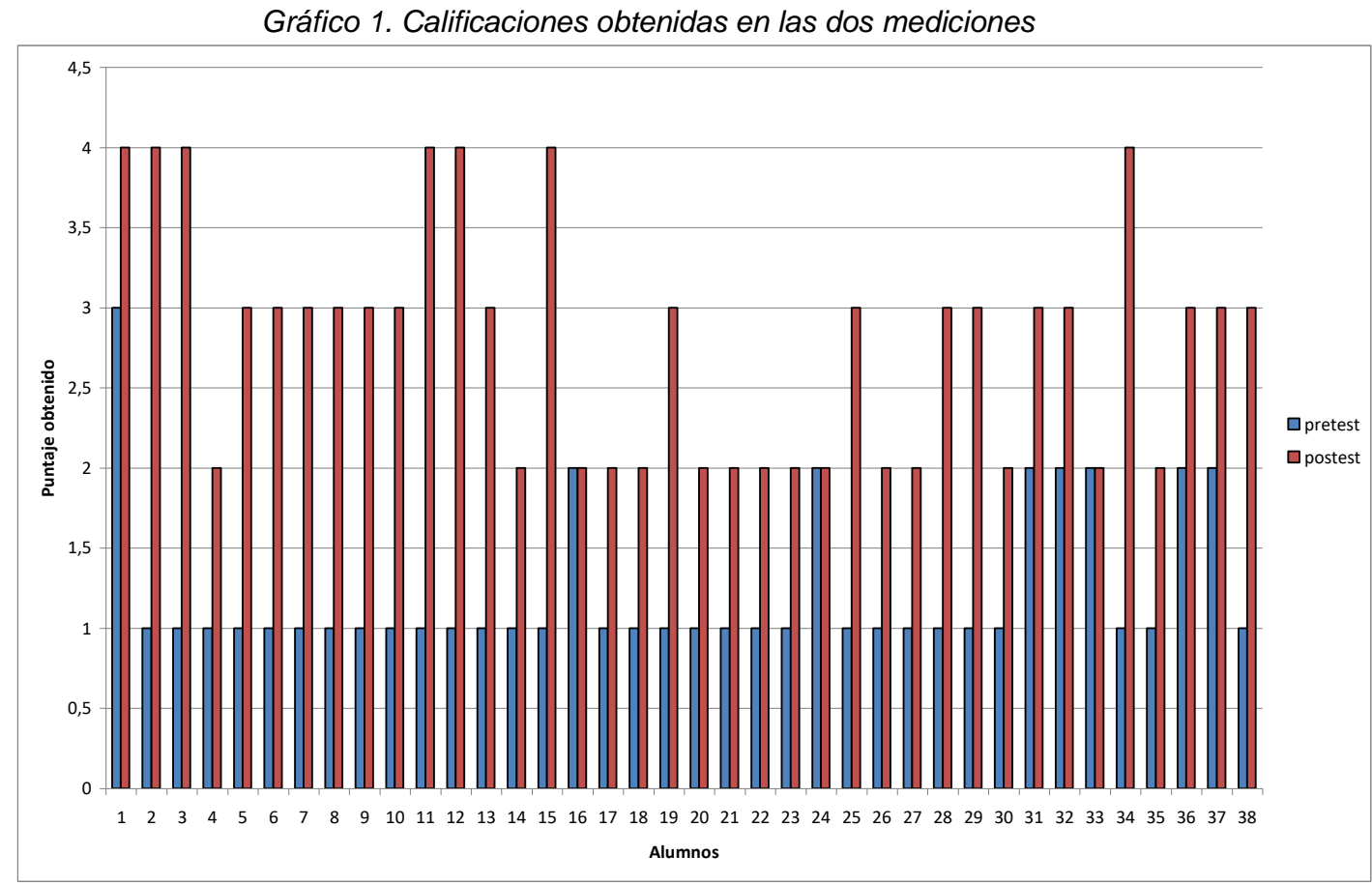

Con la finalidad de determinar estadísticamente el nivel de significancia de los resultados obtenidos, se empleó una distribución para muestras relacionadas aplicadas a un mismo grupo en dos momentos. Se usaron como variables relacionadas el pretest y el postest de la intervención, y se consideraron las siguientes:

Hipótesis nula (Ho): Las diferencias entre el pretest y el postest se deben al azar.

Hipótesis alternativa $\left(\mathrm{H}_{1}\right)$ : Las diferencias entre el pretest y el postest se deben a la intervención realizada.

Se utilizó la Prueba de Kolmogorov-Smirnov para una muestra (K-S) para determinar si los datos presentaban normalidad (Tabla 5). Se observó una mayor dispersión de los datos en la segunda medición (desviación estándar), tendencia que coincide con la idea de que hubo un cambio.

En la diferencia, el $\mathrm{P}$ valor es significativo, y por ser menor a 0,05 se pudo afirmar que los datos no tenían una distribución normal.

Tabla 5. Estadísticos de la prueba de conocimiento

\begin{tabular}{|l|l|l|l|}
\hline & Antes & Después & Diferencia \\
\hline Prueba de normalidad. Z (K-S) & 2,929 & 1,549 & $\mathbf{1 , 6 3 9}$ \\
\hline Valor de significación. P Valor & 0,000 & 0,016 & $\mathbf{0 , 0 0 9}$ \\
\hline Media & 1,24 & 2,79 & $\mathbf{1 , 5 5}$ \\
\hline Desviación estándar. ST & 0,49 & 0,74 & $\mathbf{0 , 8 6}$ \\
\hline
\end{tabular}


Por lo tanto, se optó por la prueba de rangos de Wilcoxon para dos muestras relacionadas (prueba no paramétrica), la cual arrojó un valor W calculado (Z) de -5,261 y cuyo Valor de $P$ fue de 0,000 . La probabilidad de error fue de $1,43 \mathrm{e}-7$, la cual se consideró baja. Por lo tanto el $P$ valor, menor a 0,05, favoreció la hipótesis alternativa de que las diferencias entre el pretest y el postest se debieron a la intervención realizada.

Sin embargo, ningún estudiante alcanzó la nota máxima. Se apreciaron errores de razonamiento y poco aprovechamiento de las ventajas de contar con la hoja de cálculo durante la prueba. Se buscó, entonces, lograr mejoras en cuanto al compromiso, elaboración y comunicación por parte de los educandos. Que el estudiante concienciar la importancia de trabajar para superar las deficiencias (Schlechty, 2002, citado por Reigeluth, 2012). Para ello, se resolvió la prueba, nuevamente, en clase, mediante discusiones dirigidas. Cada alumno insertó comentarios en la hoja de cálculo, en los cuales razonaba su respuesta, con apoyo del libro electrónico (Figura 3).

Este procedimiento implicó, para el estudiante, desarrollar una redacción propia, diferenciar maneras de resolver problemas, responder a las preguntas de desafío del docente, proponer soluciones, escuchar las propuestas de los compañeros y lograr consenso de ideas, lo cual supuso demostrar públicamente su nuevo conocimiento o habilidad (Reigeluth, 2012).

Para el docente, implicó proveer realimentación cara a cara, estimular la discusión grupal y redirigir el proceso, con el fin de promover la elaboración verbal y el razonamiento. De esta manera, se generó un cambio de ritmo y una sensación de formalidad, en la que el profesor observaba, desde una posición de control, las interacciones que tuvieron lugar. Los educandos, por su parte, respondieron de manera positiva a unas condiciones similares a las de la clase tradicional, y mostraron interés por mejorar el desempeño.

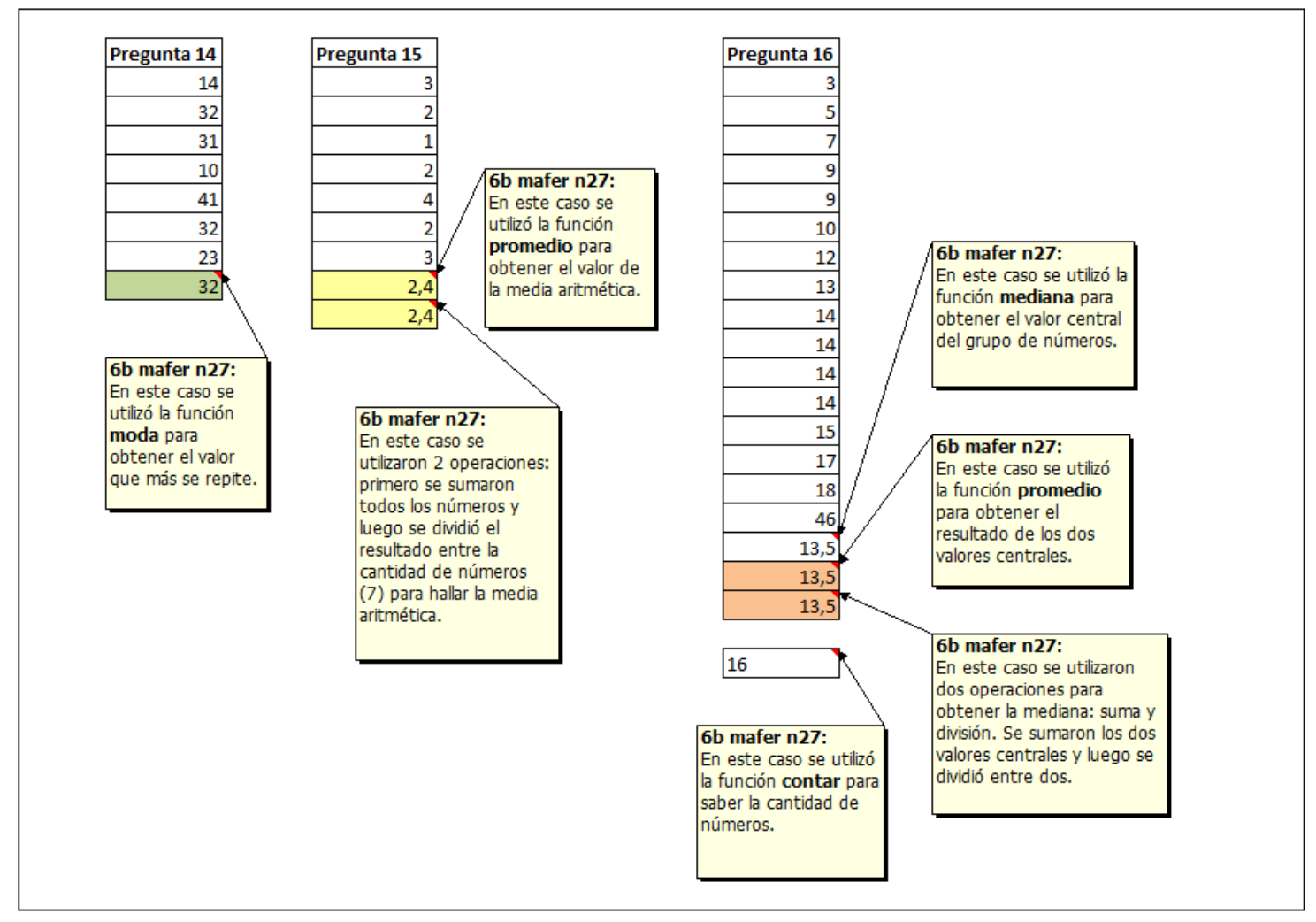

Figura 3. Ejemplo de mapa de comentarios 
El instrumento, distribuido en línea, fue completado por veintiún estudiantes (55,3\%). Los resultados indicaron una valoración positiva del libro electrónico, el cual consideraron más fácil de usar en comparación a un libro impreso. Hubo, además, una opinión favorable del grupo con respecto al desempeño del docente.

\section{DISCUSIÓN Y CONCLUSIONES}

Los resultados del diagnóstico, correspondiente a los problemas que afectan la enseñanza de la hoja de cálculo (objetivo 1), se pueden describir de acuerdo a cuatro categorías emergentes. Acerca de los medios didácticos digitales: había desconocimiento, sobre todo, en cuanto a la oferta de herramientas de creación, por lo cual se consideraban difíciles de producir. Con respecto a los textos de informática: poco adaptados al nivel evolutivo de los niños y carentes de un enfoque teórico claro. En cuanto al desempeño de los estudiantes: falta de motivación, contexto familiar poco estimulante, tendencia hacia la memorización y poco razonamiento. Sobre la percepción del libro electrónico: para el año 2013 todavía existía una línea no muy clara entre lo que era un documento PDF, un libro impreso escaneado y un libro electrónico interactivo, por lo que se percibía como un formato en evolución. Hoy quizás las diferencias están más definidas.

Se diseñó, entonces, un libro electrónico que pretendió abordar los problemas detectados (objetivo 2). Se observó y registró el desempeño de los estudiantes durante la interacción con el medio, lo cual supuso exponer al educando a la necesidad de resolver problemas mediante el seguimiento de instrucciones y el empleo de la lectura no lineal, ya que debieron releer fragmentos del texto en lugar de acudir al docente, así como elaborar verbalmente para llegar a consensos y soluciones entre pares. En este sentido, el docente ejerció un rol de observador y mediador del proceso. Atento a canalizar las dudas mediante aporte de claves para buscar en el libro electrónico y preguntas de desafío que facilitaran la reflexión por parte del educando (objetivo 3).

Se verificaron cambios que tuvieron lugar luego de aplicar el libro electrónico (objetivo 4). En lo cuantitativo, la relación pre-test y pos-test mediante pruebas estadísticas sugieren que los cambios obedecen a la intervención realizada, antes que al azar. En el aspecto cualitativo, se evidenciaron mejoras en el conocimiento procedimental, empleo de la lectura no secuencial y en la habilidad para desplazarse entre fragmentos de texto. Hubo una evolución en el desempeño con tendencia hacia la mejora durante la mayor parte del período $y$, en general, cambios positivos en la manera de interactuar con un material digital nuevo.

Resolver la prueba de conocimiento nuevamente, mediante una estrategia con base en el trabajo grupal y la elaboración verbal, tuvo como motivación primordial el compromiso de estudiar, comprobar y cuestionar el propio modo de enseñar; una actividad orientada a mejorar la práctica docente (Restrepo, 2002). Esto se vincula con los planteamientos sobre acción reflexiva basados en la evaluación, realimentación y reorientación de los procesos de enseñanza que proponen Stenhouse (1998) y Elliot (1994), y cuyo propósito es promover la práctica del rol docente-investigador.

Se pudo observar que los estudiantes adoptaron, con relativa facilidad, el procedimiento de generar notas a partir del intercambio verbal, lo cual sugiere que desarrollaron reglas de producción, reflexión, explicación y búsqueda de soluciones (metacognición), lo que es coherente con el modelo de Anderson (1980). 
Se observó que, con el paso de los días, lograban afinar su habilidad para desplazarse entre fragmentos de texto, lo cual corrobora la opinión de que la lectura en soporte electrónico es fragmentaria, y supone nuevas maneras de organizar la información, lo que quizá derive en una capacidad lectora menos intensiva y reflexiva, pero más asociativa y multimedia, tal como lo sugiere Núñez (2012).

Se constató que el libro electrónico puede generar cambios que hablan de su potencialidad como instrumento didáctico, por lo que se recomendó indagar acerca de su aplicabilidad en otras asignaturas. Se suscribe, entonces, la opinión de Reinhardt, (citado por Anzola, 2001), acerca de que las nuevas tecnologías en educación incrementan la productividad del estudiante, gracias a que impulsan un cambio cualitativo en la naturaleza del aprendizaje al promover el pensamiento creativo y la curiosidad, actitudes fundamentales para la innovación y la originalidad en el trabajo intelectual.

\section{ENLACES}

- Instrumentos de recogida de datos

- Formatos de validación

- Ficha técnica del trabajo de grado y de la propuesta

- Trabajo de grado maestría

- Una versión online del libro electrónico se encuentra disponible en la red.

- $\quad$ Presentación sobre el trabajo de grado

\section{REFERENCIAS BIBLIOGRÁFICAS}

Anzola, M. (2001). Impacto de las nuevas tecnologías en un proceso de reinserción académica de estudiantes excluidos del sistema escolar. Revista Educere, 5 (15), pp. 307-310. Recuperado de http://www.redalyc.org/html/356/35651507/

Arias, M., López, A. y Rosario, H. (2002). Metodología Dinámica para el Desarrollo de Software Educativo. En III Conferencia Internacional sobre Educación, Formación y Nuevas Tecnologías. Valencia, España. Recuperado de https://goo.gl/2f0CQ7

Calzadilla, M. (2002). Aprendizaje colaborativo y tecnologías de la información y la comunicación. Revista Iberoamericana de Educación, 24 (1). Recuperado de https://goo.gl/QDtzMR

Canchignia, X. (2011). Libro electrónico de openoffice.org Calc para mejorar el aprendizaje del módulo de hoja electrónica de cálculo en el segundo año de bachillerato del Instituto Tecnológico Victoria Vásconez Cuvi, Latacunga. Ecuador. Recuperado de https://goo.gl/vmZ4ml

Casado, R. (2013). Ebooks y el paradigma tecnológico de la educación. Razón y Palabra, 1 (49), pp. 138-143. Recuperado de https://goo.gl/hpEcQd

Elizondo, F. (2006), e-Book. Revista Ingenierías FIME-UANL, 9 (32). Recuperado de www.ingenierias.uanl.mx/32/32 editorial.pdf

Elliot, J. (1994). La investigación-acción en educación. Madrid: Morata.

Hurtado, J. (2012). Metodología de la investigación: Guía para una comprensión holística de la ciencia. Bogotá: Ciea-Sypal. 
Lai, H., y Li, M. (2013). Un studio del comportamiento de estudiantes universitarios en el uso de libros electrónicos en Hong Kong. Knowledge Management \& E-Learning, 5 (4), pp. 455467. Recuperado de https://goo.gl/hwa6OU

Louis de Vivas, M. (1994). Investigación cualitativa. Manual de para la recolección y el análisis de la información. Caracas: El Juego Ciencia.

Marquès, P. (2011). Medios didácticos y recursos educativos: componentes, tipología, funciones, ventajas asociadas a su utilización. Barcelona: Universidad Autónoma de Barcelona. Recuperado de http://peremarques.pangea.org/medios.htm

Miranda, N., Pirez, Y. y García, J. (2012). La tarea integradora y el aprendizaje en los estudiantes de secundaria básica. La Habana: Universidad de Ciencias Pedagógicas José Martí. Recuperado de http://www.rimed.cu/medias/evaluador/Ines-Maria.pdf

Núñez, P. (2012). Crítica de libros: Gutenberg 2.0: la revolución de los libros electrónicos. Revista Española de Documentación Científica, 35 (1), pp. 191-194. Recuperado de http://goo.gl//U7R5V

Piaget, J. (1991). Seis estudios de psicología. Barcelona: Labor.

Poggioli, L. (2009). Estrategias de aprendizaje: una perspectiva teórica. Caracas: Fundación Empresas Polar.

Ramos, P. y Moreira, J. (2013). e-Book: un dispositivo pedagógico en la enseñanza y aprendizaje de biología y geología - un caso de estudio con estudiantes de onceavo grado. International Journal of Secondary Education, 1 (6), pp. 53-63. Recuperado de http://article.sciencepublishinggroup.com/pdf/10.11648.j.ijsedu.20130106.12.pdf

Reigeluth, C. (2012). Teoría instruccional y tecnología para el nuevo paradigma de la educación. RED Revista de Educación a Distancia. 11 (32). Recuperado de http://www.um.es/ead/red/32

Restrepo, B. (2002). Una variante pedagógica de la investigación-acción Educativa. Revista Iberoamericana de Educación, 30 (1). Recuperado de http://rieoei.org/inv edu12.htm

Stenhouse, L. (1998). La investigación y el desarrollo del currículum. Madrid: Morata.

\section{INFORMACIÓN SOBRE EL AUTOR}

\section{Marío José Contreras Rodríguez \\ Instituto Universitario Pedagógico Monseñor Rafael Arias Blanco}

Profesor tutor del Instituto Universitario Pedagógico Monseñor Rafael Arias Blanco (IUPMA), asociado a la Universidad Pedagógica Experimental Libertador (UPEL). Magister en Educación Técnica por la UPEL. Profesor en informática por el IUPMA, con experiencia en todos los niveles del sistema educativo venezolano. Miembro de la Comisión Evaluadora de Proyectos de Trabajos de Grado del IUPMA. Desarrolla la línea de investigación Tecnología Aplicada a la Educación Técnica y a la Educación para el Trabajo. Ha participado como miembro del comité organizador y ponente del Congreso Regional Anual de Investigación (CRAI) en Caracas. Participa en proyectos relacionados con las TIC en el ámbito educativo, el diseño de medios didácticos digitales y la gestión del conocimiento.

www.educapuntes.blogspot.com

https://www.linkedin.com/in/mario-contreras-22481851/

\section{(cc) EY-NC}

Los textos publicados en esta revista están sujetos a una licencia de Reconocimiento 4.0 España de Creative Commons. Puede copiarlos, distribuirlos, comunicarlos públicamente y hacer obras derivadas siempre que reconozca los créditos de las obras (autoría, nombre de la revista, institución editora) de la manera especificada por los autores o por la revista. La licencia completa se puede consultar en:Licencia Creative Commons Atribución-NoComercialCompartir por igual 4.0 Internacional. 